\title{
KRAS NM_004985.3:C.38_39delGCinsTG
}

National Cancer Institute

\section{Source}

National Cancer Institute. KRAS NM 004985.3:C.38 39de/GCinsT G. NCI Thesaurus. Code C98422.

A complex substitution where the nucleotide sequence at positions 38 through 39 of the coding sequence of the KRAS gene has changed from guanine-cytosine to thymineguanine. 